\title{
Confocal imaging of the fundus using a scanning laser ophthalmoscope
}

\author{
W H Woon, F W Fitzke, A C Bird, J Marshall
}

\begin{abstract}
A confocal scanning laser ophthalmoscope (cSLO) was used to examine the effects of confocal optics on the image of the human fundus in vivo. Patients from a retinal clinic and a glaucoma clinic were examined using the cSLO in the confocal mode. A degree of optical sectioning could be achieved, and the results agree with a best axial resolution of $300 \mu \mathrm{m}$ measured in a model eye. The main advantage of using a confocal system was found to be the improved contrast of the images. This improved the resolution of structures such as the lamina cribrosa and optic disc drusen which are seen in low contrast in conventional images. The improved contrast of the confocal images is partly achieved by excluding light which has been scattered within the plane of focus. Structures which multiply scatter light will become less visible with confocal optics and hard exudates were found to be an example of such a structure. The cSLO and the fundus camera are seen as complementary instruments rather than as alternatives for imaging the fundus. It is envisaged that confocal imaging will enable details of the fundus to be revealed which are at present not seen in conventional images.
\end{abstract}

(BrF Ophthalmol 1992; 76: 470-474)

Confocal imaging is an established technique for obtaining high resolution images in the laboratory. ${ }^{1}$ In vivo confocal imaging of the human fundus can now be used by the ophthalmologist with the introduction of a commercially available confocal scanning laser ophthalmoscope (cSLO). The scanning laser ophthalmoscope uses a focused laser beam to scan over the area of the fundus to be imaged..$^{2-4}$ At any instant only a small spot on the fundus is illuminated, and the light returned from this spot inside the eye determines the brightness of a corresponding point (pixel) on the TV monitor (Fig 1). If the laser is made to scan over successive points on the fundus then an image can be built up as an array of pixels on the TV monitor. This is done by

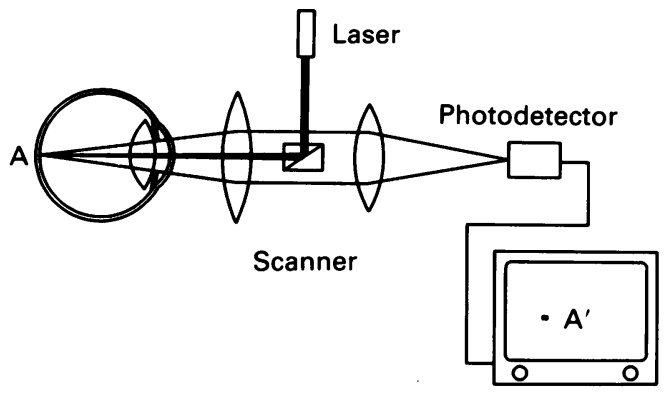

Monitor using a spinning mirror and an oscillating mirror, and the laser beam is made to scan in a raster fashion across the fundus. The angle through which the laser beam is scanned determines the area of the fundus that is scanned and therefore the field of view. A large scanning angle will give a large field of view and therefore a low magnification. The Rodenstock 101 cSLO can operate in a 20 degree field of view mode or a 40 degree field of view mode.

If the photodetector in the SLO accepts all the light leaving the eye under examination, then the image is said to be non confocal (Fig 2, top). Non-confocal images suffer from loss of contrast due to light which is returned to the detector after being scattered by media opacities or after multiple scattering in the fundus. To improve

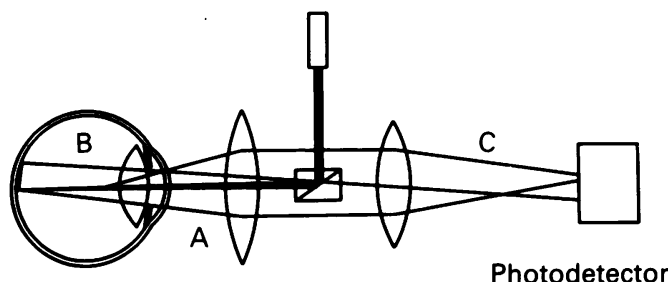

Non-confocal mode

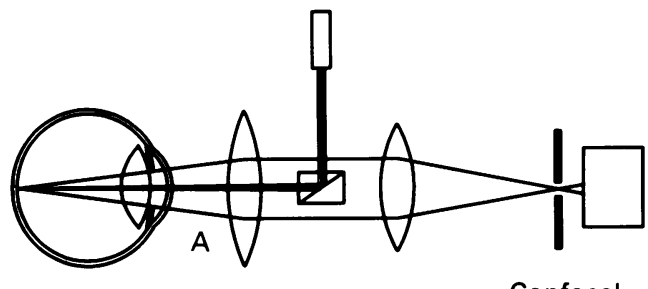

Confocal mode

Confocal aperture

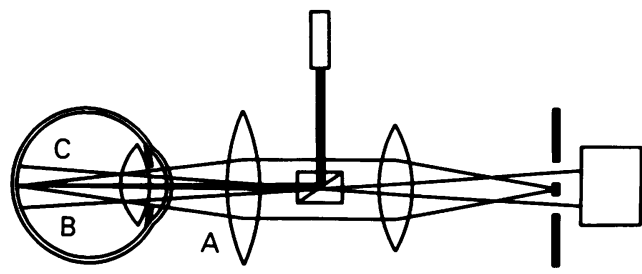

Dark field mode

Annular aperture

Figure 2 Top: non-confocal mode. The photodetector accepts a high proportion of the light scattered back from the eye. Ray $A$ is light scattered from the laser focus on the retina. $R a y B$ is light which has been side scattered from the laser focus before being rescattered out of the eye. Ray C is light which has been scattered from the optical media. Rays $B$ and $C$ do not directly come from the laser focus and reduce the contrast of the image of the fundus. Middle: confocal mode. A pinhole (confocal aperture) placed conjugate to the laser focus at the fundus ensures that only rays of light $(A)$ directly

scattered back from the laser focus pass to the photodetector. Bottom: dark field mode. An annular aperture blocks out rays of light $(A)$ directly scattered back from the laser focus. Rays of light $(B, C)$ which have been side scattered from the laser focus before being rescattered out of the eye can pass through to the photodetector. 
Figure 3 Top: the confocal aperture $(C)$ ensures that only light from a small volume or voxel $(V)$ reaches the detector $(D)$. Bottom: point $A$ is not conjugate to the confocal aperture and most of the light from point $A$ is blocked by the confocal aperture. Light from structures outside the plane of the voxels will not contribute to the image.
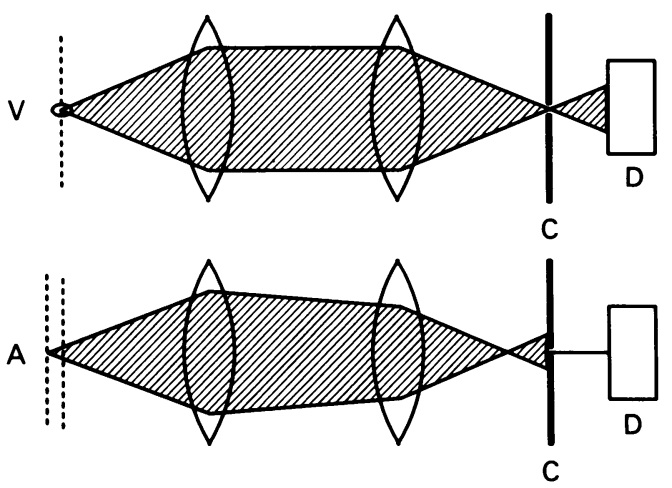

image contrast the detector should only receive light that is scattered back directly from the laser focus on the fundus. This is achieved by placing a pinhole, which is conjugate to the laser focus, in front of the detector ${ }^{34}$ (Fig 2, centre). The pinhole is called a confocal aperture and the image so obtained is said to be confocal. The instantaneous volume of tissue from which back scattered light is accepted by the confocal aperture is called a voxel (Fig 3, top). The size of the confocal aperture determines the degree of confocality of the image, a small confocal aperture gives rise to a small voxel and a highly confocal image. ${ }^{5}$ In a confocal system, the detection unit is instantaneously accepting light from a single point on the fundus, and the detection system must be made to scan in synchrony with the laser illumination so that the detection system and illumination system are directed at the same point on the fundus. This is achieved by the double scanning of the cSLO which uses the same scanning system in reverse directions for the illumination and detection systems. ${ }^{34}$

In the confocal mode, an image is built up of light scattered back from a layer of voxels, and the image is thus taken from a thin section of equal thickness to the depth of a voxel. In this way an optical section is produced, as light from structures outside the plane of the voxels will not contribute to the image (Fig 3, bottom). This may be contrasted with conventional imaging where objects outside the depth of field contribute a blurred image and can degrade the image of objects within the depth of field. The thickness

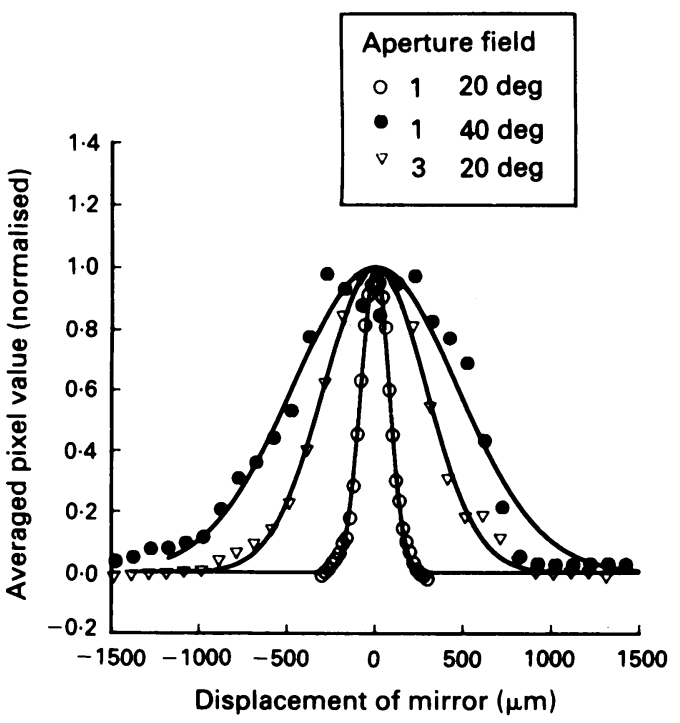

of the optical section will depend on the confocality of the image. A smaller confocal aperture will give a narrower optical section within the limits imposed by the optics of the human eye. ${ }^{67}$ The axial position of the optical section may be altered by altering the plane that is conjugate to the confocal aperture. In the Rodenstock 101 cSLO, this may be done by using the ametropic corrector. Adding negative power to the ametropic correction will move the plane of focus deeper in the eye, and vice versa. The ability of confocal imaging to separate structures in different optical sections gives it axial resolution, though imaging an optical section deep to the surface of an object is only possible for transparent or translucent objects.

The cSLO can be used in a mode which produces an image which is effectively the image produced by subtracting the confocal image from the non-confocal image. This mode is called the dark field mode and is produced by using an annular aperture in place of the pinhole confocal aperture. ${ }^{3}$ The annular aperture blocks out light directly scattered back from the laser focus while allowing light which has been rescattered from an area adjacent to the laser focus to pass through (Fig 2, bottom). Thus the dark field mode detects multiply scattered light, and objects which have many scattering centres will appear bright in this mode.

The images from the presently available cSLO are monochromic, and differences in spectral information are lost. In addition, a confocal image differs from a conventional monochromatic image because the contrast in a confocal image arises mainly from differences in reflection and refraction rather than differences in absorption of the incident light.$^{8}$ This means that the confocal image does not show the same variation with wavelength that is found with monochromatic fundus camera images. In a confocal image blood vessels show up well using red illumination, while this is not true for conventional monochromatic images. This is particularly important as the red HeNe laser is most commonly used in cSLOs.

The visibility of a structure depends on its contrast with its surroundings. Thus, although the resolution of the cSLO may be less than the resolution of a conventional fundus camera for structures which are seen in high contrast, the cSLO may be able to resolve structures which cannot be resolved by conventional imaging because of poor contrast.

We have used a commercially available cSLO to assess the value of confocal imaging of the fundus. The axial resolution was measured in a model eye, and confocal images were obtained from patients.

\section{Methods}

The axial resolution of the cSLO was measured using a method that has been previously described. ${ }^{9}$ A model eye was used which consisted of a $16 \mathrm{~mm}$ lens and a micrometer mounted mirror. The pixel brightness of the cSLO image of the mirror was measured for a range of axial positions of the mirror.

Fifty one patients were examined using a
Figure 4 Axial resolution of the CSLO measured in a
model eye. The best axial resolution is achieved using confocal aperture 1 in the 20 degree field of view mode. 
cSLO. The patients were recruited from a retinal clinic and a low tension glaucoma clinic. Patients from the retinal clinic were already dilated with tropicamide $1 \%$ and phenylephrine $10 \%$, while patients from the low tension glaucoma clinic were dilated with tropicamide $1 \%$ if required. The patients were examined with a Rodenstock $101 \mathrm{cSLO}$ using the $\mathrm{HeNe}$ laser alone. Images were recorded using a U-matic video recorder (Sony VO-5850P).

Optical sectioning of the live fundus was performed using the 20 degree field of view and confocal aperture 1. The axial position of the optical section was varied by altering the setting of the ametropic corrector.

A range of pathological conditions was examined using the confocal mode of the cSLO. The ametropic corrector was used to correct for ametropia, and to focus on the structure of interest. Images were produced using a range of confocal apertures and both the 20 degree field and 40 degree field of view mode. Images were compared with colour slides obtained using a fundus camera. In selected patients, enhanced confocal images were also produced by computer averaging over 16 frames. This was done using an IBM AT personal computer with a FG100 frame grabber. The program was supplied by the Eye Research Foundation, Boston. The frames were aligned manually before averaging. No other computer enhancement was performed on the images. Hard copies of averaged images fundus camera (right) image of disc drusen. The nodular structure of the drusen is apparent in the confocal cSLO image.

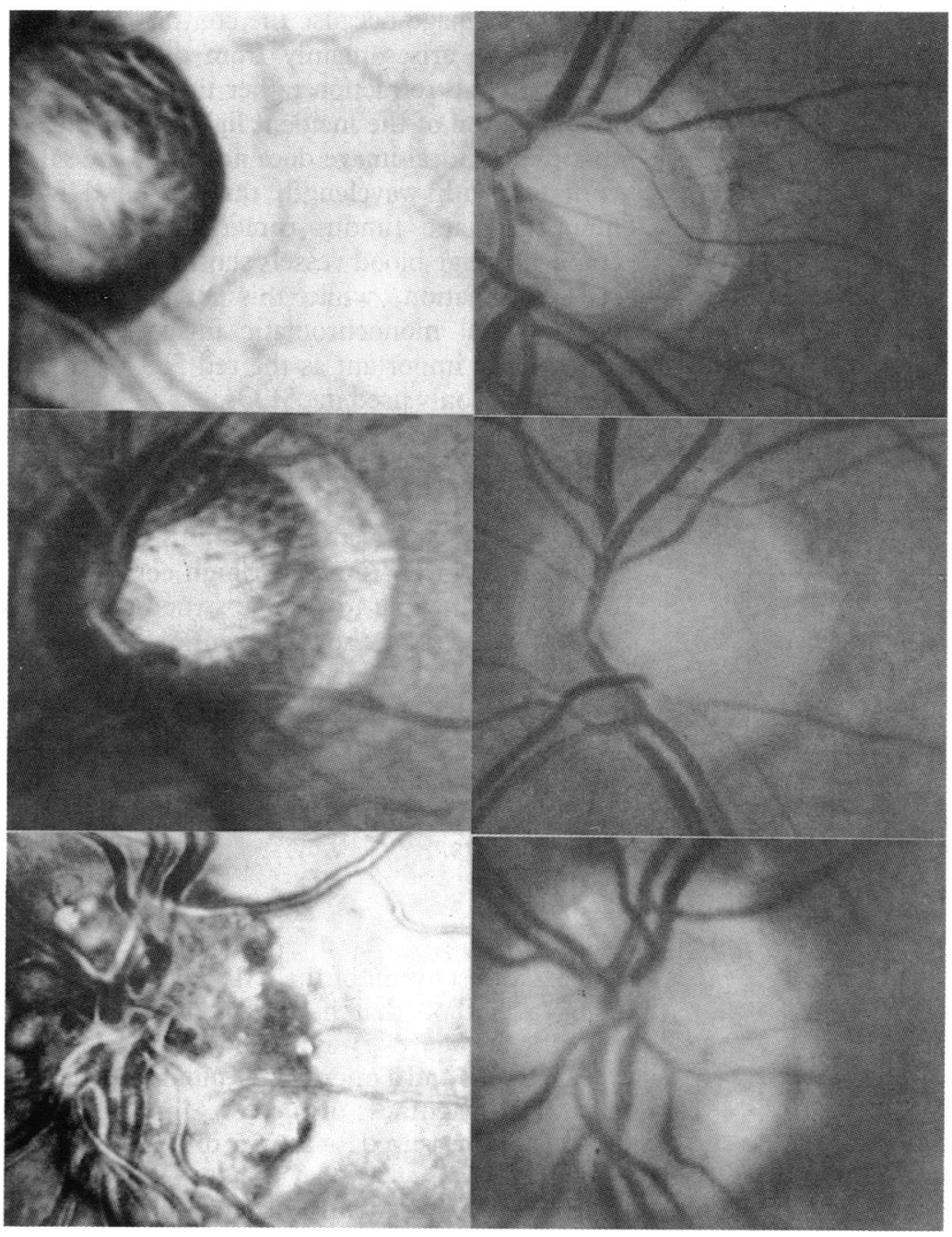

were obtained by photographing (FP4 film) the video monitor (Sony PVM 1440QM).

\section{Results}

The cSLO was not difficult to learn to use. The level of retinal irradiance was comfortable, with several patients remarking spontaneously that they preferred the cSLO to the fundus camera. It was found that pupillary dilatation was helpful when using the cSLO in the 20 degree field of view mode, but was not necessary when using the 40 degree field of view.

The axial resolution of the cSLO in the model eye is shown in Figure 4. The axial resolution is measured from the points where the brightness of the image of the mirror has fallen by a half. The best axial resolution (full width at half maximum, FWHM) is $300 \mu \mathrm{m}$ for the 20 degree field of view and the smallest confocal aperture (aperture 1). In practice, when using confocal aperture 1 and the 20 degree field of view, the image quality suffered from insufficient light levels, even with maximum illumination. Light levels using larger confocal apertures in the 20 degree field mode or confocal aperture 1 in the 40 degree field mode were found to be sufficient.

Optical sectioning was most easily demonstrated in imaging the disc in patients with low tension glaucoma. It was possible to obtain high quality images of the lamina cribrosa in all 10 patients with low tension glaucoma by focusing deep to the retina (Fig 5, top). The lamina cribrosa was visible using conventional ophthalmoscopy, but was difficult to photograph using a fundus camera. In two patients it was possible to image the lamina cribrosa underlying the neuroretinal rim using confocal optics (Fig 5, centre). Improved contrast was also demonstrated in a patient with optic nerve head drusen and a patient with an optic disc melanocytoma (Fig 5, bottom and Fig 6, top).

The improvement in contrast with confocal imaging is best demonstrated in imaging the retinal vessels. The contrast could be improved by reducing the size of the confocal aperture (Fig 7).

Vitreous floaters were often seen using the cSLO. In one patient with low tension glaucoma, a vitreous discontinuity with macular attachment could be demonstrated (Fig 6, bottom).

In three patients with hard exudates, it was found that the exudates became less visible using confocal imaging, and that the exudates were well seen using the dark field mode (Fig 6, centre, and Fig 7).

It was found that, subjectively, a single frame image captured from the video tape was not of comparable quality to the dynamic video recording. Computer averaging over 16 frames produced images that were subjectively of comparable quality to the dynamic video recording. No new details were seen in the averaged images.

\section{Discussion}

The cSLO provides a novel method of imaging the fundus. At present, when used purely for non-dynamic imaging, the cSLO should be considered as complementing rather than replac- 


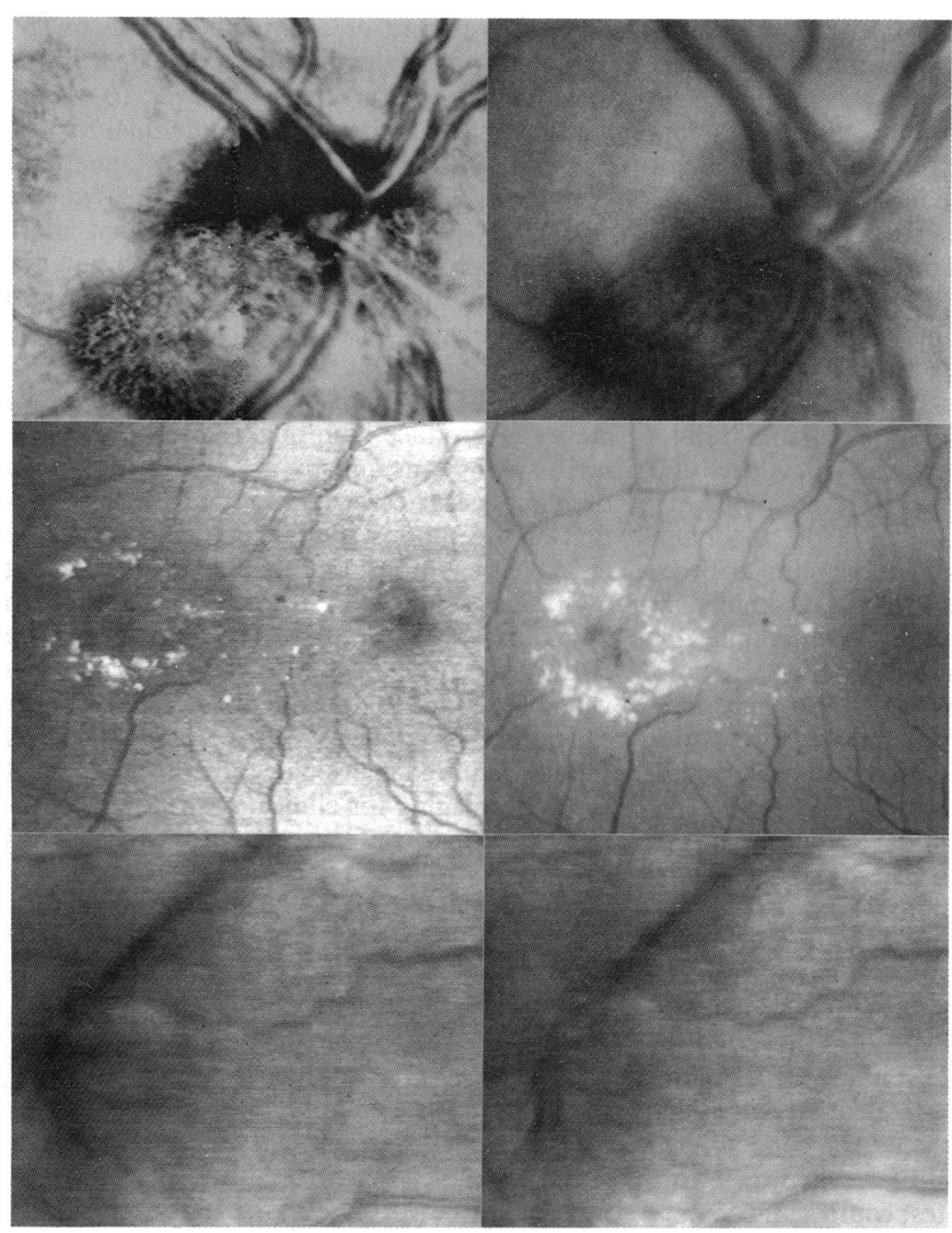

Figure 6 Top: confocal (left) and fundus camera (right) image of a disc melanocytoma. The contrast of structures within the melanocytoma is increased in the confocal image. Centre: confocal (left) and fundus camera (right) image of diabetic exudates taken on the same day. The exudates appear to be more extensive in the fundus camera image. Bottom: confocal images, taken a short time apart, of a vitreous discontinuity in a patient with low tension glaucoma. The vitreous discontinuity appears to be fixed at the macula. ing the functions of a fundus camera. The fundus camera produces an image that is equivalent to that observed by the clinician using an ophthalmoscope. The confocal image produced by the cSLO changes the appearance and visibility of structures from that in a conventional image. This allows some, but not all, structures to be better seen in a confocal image.

The scanning laser ophthalmoscope has been developed to image the fundus without the need for mydriatics. ${ }^{3}$ This would be an advantage when there is a large through-put of patients such as in screening programmes. In the cSLO, this advantage was only consistently found when using the 40 degree field of view. Using the 20 degree field of view, a mydriatic was found to be helpful. This is due to the increase in laser beam diameter at the pupil, from $1 \mathrm{~mm}$ to $2 \mathrm{~mm}$, when changing from the 40 degree to the 20 degree field of view. ${ }^{10}$ Thus, in the 20 degree field of view mode, a larger area of the pupil is used for the illumination and a pupil size greater than $2 \mathrm{~mm}$ is required to obtain a view of the fundus. The 20 degree field of view mode gives a more confocal image, and in this study the minor inconvenience of using a mydriatic was accepted in order to produce a more confocal image. The cSLO has been shown to give a degree of optical sectioning. The best axial resolution of the cSLO was found to be $300 \mu \mathrm{m}$ (FWHM) in a model eye. This agrees with the results of other investigators. ${ }^{10}$ The optical sectioning demonstrated in the live eye was probably of the same order and would be sufficient to section the retinal features from the lamina cribrosa in patients with deep cups. However, most other clinically important features in the fundus lie within too narrow a plane for them to be optically sectioned from each other. Although an axial resolution of $300 \mu \mathrm{m}$ is disappointing in terms of optical sectioning of the fundus, it does improve contrast by avoiding flare from the ocular media. Contrast is also improved in the confocal mode by avoid-
Figure 7 SLO images of a patient with diabetic maculopathy taken using the 40 degree field of view. Top left: confocal aperture 3. Top right: confocal aperture 1. Bottom left: dark field image. Bottom right: non-confocal image. The blood vessels are seen in higher contrast in the confocal images while the
exudates are less visible in the confocal images.

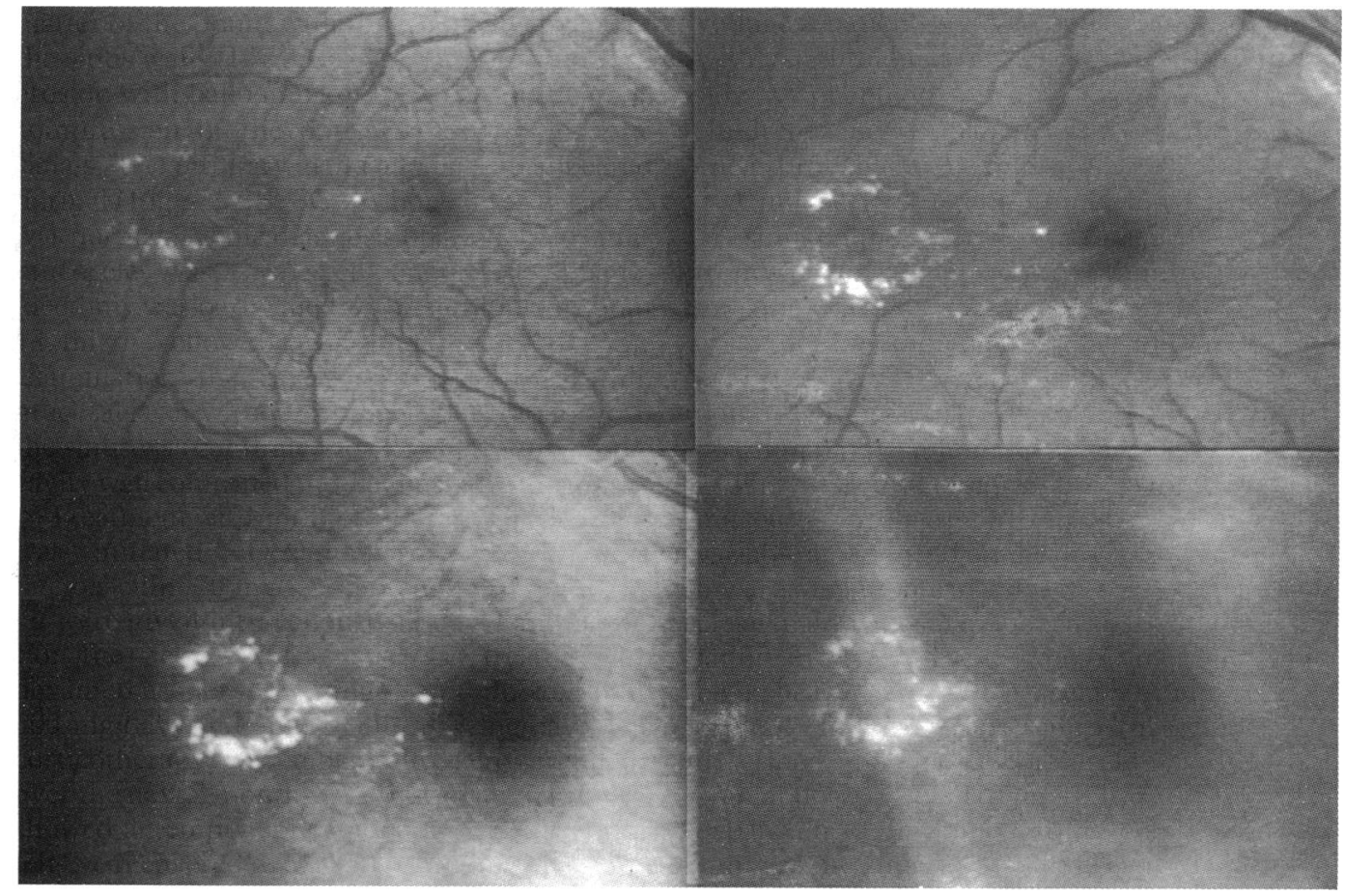


ing multiply scattered light from the fundus. It was found that the improved contrast of confocal imaging is of particular value in improving the resolution of structures that are seen in low contrast in conventional fundus camera images (Figs 5 and 6). The avoidance of light scattered from the nerve fibres in the optic nerve head allowed optic nerve head drusen to be imaged as discrete nodules. Similarly in two patients, it was possible to image the lamina cribrosa underlying the neuroretinal rim. This was probably due to a relatively thin neuroretinal rim in these two patients, while in the majority of patients scattering of light by the neuroretinal rim caused too much attenuation of the laser illumination. For similar reasons, it was not possible to obtain multiple optical sections of the lamina cribrosa even though the lamina cribrosa is known to extend over $1 \mathrm{~mm}$. Nonetheless, the ability to image the surface of the lamina cribrosa will be of benefit in examining the hypothesis that optic nerve head damage from raised intraocular pressure is related to the structure of the lamina cribrosa. ${ }^{112}$

The improved contrast of the confocal images is gained at the cost of the visibility of structures which multiply scatter light. These structures are well imaged using a conventional fundus camera, and this demonstrates the complementary nature of the cSLO and the fundus camera. Hard exudates are clearly visible in the dark field mode of the cSLO (Fig 7). They must therefore multiply scatter light. Their apparent extent in a confocal image is less than in a conventional image, and it is important that, when reviewing patients with hard exudates, confocal images are not confused with conventional images. However it would be reasonable to compare directly an SLO image produced using the non-confocal mode with a conventional fundus camera image.

The cSLO was found to image vitreous discontinuities in higher contrast than a fundus camera. The vitreous disturbances were usually seen as shadows floating across the screen. However, in one patient, the vitreous was found to be attached around the fovea (Fig 6, bottom). The dynamic image from the cSLO showed movement of the area of abnormal vitreous with one end remaining attached around the fovea. Further observations on vitreous attachments using the cSLO may help to clarify the role of the vitreous in the development of macular holes. ${ }^{13}$

The subjective quality of the confocal images was good for the dynamic cSLO image, while single frame images captured from the dynamic image contained noise that was not evident in the dynamic image. This difference is probably due to the ability of the human brain to average over successive frames of a dynamic cSLO image. The quality of a dynamic image could be approximated by computer averaging over 16 frames with manual alignment of the individual frames. No extra details were obtained by computer averaging that could not be seen in the dynamic recording. However it is possible that more accurate alignment of the images by computer or other forms of computer enhancement could provide greater resolution of details of the fundus.

The cSLO has created most interest by its ability to produce high quality dynamic fluorescein angiograms and by its ability to perform pyschophysical tests with simultaneous visualisation of the fundus. Our results show that the cSLO has an important role to play in providing high contrast images of structures that are seen in low contrast using conventional techniques. The cSLO, by providing confocal imaging, is a valuable adjunct for imaging the fundus. Further experience of confocal imaging of pathological fundi is required to evaluate fully the potential of confocal imaging for clinical use. However it is expected that confocal imaging will make a valuable contribution to clinical research.

W H Woon is the Lady Allerton Research Fellow supported by the Lady Allerton Research Fund.

F W Fitzke is supported by the Wellcome Trust, the MRC, and the National Retinitis Pigmentosa Foundation Incorporated.

The authors thank the Eye Research Foundation, Boston for supplying their software for use with the FG100 frame grabber. The authors are also very grateful to Mr T J ffytche, Mr A M P Hamilton, and Mr R A Hitchings for allowing their patients to take part in this study.

1 White JG, Amos WB. Confocal microscopy comes of age. Nature 1987; 328: 183-4.

2 Webb RH, Hughes GW, Pomerantzeff O. Flying spot TV ophthalmoscope. Appl Opt 1980; 19: 2991-7.

3 Webb RH, Hughes GW, Delori FC. Confocal scanning laser ophthalmoscope. Appl Opt 1987; 26: 1492-9.

4 Woon WH, Fitzke FW, Chester GH, Greenwood DG Marshall J. The scanning laser ophthalmoscope. $\mathcal{F}$ Ophth Photogr 1990; 12: 17-23.

5 Inoue S. Foundations of confocal scanned imaging in light microscopy. In: Pawley JB, ed. Handbook of biological confocal microscopy. New York: Plenum. Revised edition, 1990: Chapter 1, 1-14

6 Campbell FW, Gubisch RW. Optical quality of the eye. f Physiol 1966; 186: 558-78.

7 Dreher AW, Bille JF, Weinreb RN. Active optical depth resolution improvement of the laser tomographic scanner. Appl Opt 1989; 28: 804-8.

8 Webb RH, Delori FC. How we see the retina. In: Marshall J, ed. Laser technology in ophthalmology. Amsterdam: Kugler \& Ghedini Publications, 1990: 3-14.

9 Fitzke FW, Woon H, Timberlake G, Robinson L, Marshall J, Bird AC. Optical modifications to a scanning laser ophthalmoscope for high magnification, narrow optical section imaging. Laser Light Ophthalmol 1991; 4: 7-14.

10 Plesch A, Klingbeil U. Optical characteristics of a scanning laser ophthalmoscope. SPIE 1989; 1161: 390-8.

11 Quigley HA, Addicks EM. Regional differences in the structure of the lamina cribrosa and their relation to glaucomatous optic nerve damage. Arch Ophthalmol 1981; 99: 137-43.

12 Quigley HA, Addicks EM, Green WR, Maumenee AE. Optic nerve damage in human glaucoma: the site of injury and susceptibility to damage. Arch Ophthalmol 1981; 99: 635-49.

13 Avila MP, Jalkh AE, Murakami K, Trempe CL, Schepens $\mathrm{CL}$. Biomicroscopic study of the vitreous in macular breaks. Ophthalmology 1983; 90: 1277-83. 